\title{
Baltazar Caravedo
}

2016 La energía social de la empresas B.

Lima: Universidad del Pacífico.

por Julio Gamero

jgamero@pucp.pe

Webb, J.B.; R.D. Ireland \& D.J. Ketchen (2014). Toward a greater understanding of entrepreneurship and strategy in informal economy 


\section{Introducción}

El libro objeto de la presente reseña se titula La energía social en las empresas $B$ y su autor es el doctor Baltazar Caravedo Molinari. Como conocedor de los límites de la responsabilidad social empresarial, su acercamiento a las denominadas "empresas B» (o "circulares", como las denomina) no hace sino revelar su continua reflexión en temas vinculados con la relación entre las empresas y la sociedad.

El libro ubica la reflexión en dos dimensiones: una primera en relación con el sistema y la otra con la organización/empresa. En medio de estas dos dimensiones, se trabaja la interrelación entre ambas y el impacto que tiene una forma organizacional en la viabilidad del sistema a partir de la generación de una determinada calidad de lo que denomina "energía social».

Así, el propósito del texto, según el autor, es «destacar la importancia de la producción de energía social de una organización y el impacto que puede tener en la sociedad» (p. 10). Para ello, se traza algunas interrogantes: "¿qué origina la transformación de una empresa?, ¿cómo impactan las empresas en la sociedad? y ¿cómo impactan las empresas B en la sociedad?» (p. 9).

La temática en cuestión la aborda en cuatro capítulos: en el primero, reflexiona sobre la energía social y la empresa; en el segundo, desarrolla lo concerniente a la fragmentación empresarial y la discusión sobre el valor económico y el valor social; en el tercer capítulo, desarrolla las limitaciones de la responsabilidad social empresarial; y en el último capítulo, problematiza el porqué de la necesidad de impulsar empresas B en el país.

\section{Energía social y empresa}

En este capítulo — que es el de mayor desarrollo y el que da la base sobre la que se construye todo el discurso posterior-, la reflexión se concentra en dos aspectos: el primero está relacionado con el sistema y la energía social, y el segundo, con la conectividad. En ambos, un común denominador es la incorporación de la empresa como sujeto sustantivo de la configuración del sistema y de la intensidad de la energía social que se transmite.

Un hilo conductor es la preocupación del autor por la sostenibilidad sistémica de la organización y la sociedad. Así, menciona que: "Para la adaptación a su entorno, los sistemas vivos necesitan producir una transformación en sí mismos. Y esta es la resultante del balance entre energías con diferente signo que facilitan o entorpecen su continuidad[...] La sobrevivencia y autogeneración de los sistemas depende de la capacidad para renovar energía y mantener baja entropía» (p. 14).

La denominada "energía social» es la que acaba cumpliendo el rol de coadyuvar a la sostenibilidad del sistema en tanto se la entiende como la resultante de una confrontación entre las fuerzas integradoras y las desintegradoras. En términos genéricos, expresa la resultante de la confrontación entre las energías positivas, que son aquellas que ayudan a la continuidad del sistema y a las que denomina "energías de cohesión», con las de signo negativo, que son todas las que al alentar los conflictos acaban desordenando el sistema, aumentando la entropía y poniendo en riesgo su sostenibilidad. 
Si bien no se ensaya una definición explícita de la energía social, se señala que ella es "producto de la interacción de los componentes de un sistema humano» (p. 23). Igualmente, se menciona que: «El balance de la energía social es la diferencia entre la energía positiva y la negativa. Si es positivo habrá continuidad del sistema social, predomina la energía de cohesión» (p. 23). También escribe que: "La energía social está asociada directamente a los vínculos que se establecen entre las personas» (p. 23), lo que refuerza lo explicitado con relación a su asociación con los componentes de un sistema humano y, por ello, son las acciones de los seres humanos las que originan y le dan el sentido en una u otra orientación —-positiva o negativa— a dicha energía.

En esa visión integradora de sistemas y empresas, es que se señala que no todas las formas organizacionales empresariales producen/generan la suficiente energía positiva; es decir, aquella energía que impregnará cohesión a la sociedad. Por las razones que se expondrá en los capítulos siguientes, se anticipa que son las empresas denominadas «circulares» o «B» las que aportan en dicho sentido.

Todo concepto requiere de una métrica que lo haga visible, que lo operacionalice y facilite su incorporación como indicador referente para los diversos procesos alineados con la toma de decisiones, tanto a nivel empresarial como de la política pública. De tal situación no se encuentra exento el concepto de "energía social». El autor recurre para ello a un trabajo de Chaisson (2016), donde se hace referencia a un indicador denominado "ratio de densidad de energía» (RDE, que se expresa en ergios por segundo por gramo) que permite medir la energía de los sistemas humanos. Concluye que estos últimos, al ser de mayor complejidad, acaban generando mayor energía social que el Sol, por ejemplo, en una proporción de 500000 a 2.

De otro lado, toda energía requiere de un mecanismo transmisor que la convierta en un agente dinamizador de procesos. Ello se desarrolla bajo el concepto de "conectividad", la cual "facilitará o entorpecerá el flujo de energía» (p. 25). Se recurre a la figura de los circuitos eléctricos para ilustrar la mayor o menor intensidad en la transmisión de los flujos de energía. De esta manera, se menciona la diversidad de modalidades de conectividad. De menor a mayor intensidad en el traslado de energía, se tiene: 1) de punto a punto, cuando la conexión entre diversos dispositivos opera a partir de un único canal troncal; 2) estrella, cuando se da una conexión directa de diferentes dispositivos a un centro; 3) tipo bus, cuando a partir de la presencia de una sola vía se conectan diversos dispositivos; y 4) tipo malla y tipo árbol, donde el primero toma la figura de un nodo conectado a todos los nodos y el segundo de redes de estrellas. En la medida en que la conectividad involucre a una mayor cantidad de puntos —es decir, cuando predomine la forma tipo malla o árbol—, el flujo de energía generado será de mayor intensidad.

La reflexión sobre conectividad, sistemas y energía social las aplica a la caracterización que realiza de los sistemas sociales. Así, Caravedo distingue tres sistemas: tradicional, moderno y tecnológico. El primero lo vincula con la modalidad de conectividad calificada de punto a punto. Ello implica que en dicho sistema hay menor intensidad en la generación de flujos de energía. En el sentido opuesto, el sistema tecnológico se caracteriza por una conectividad más compleja y profunda en la que los tipos malla y árbol resultan predominantes. Los tres sistemas no son necesariamente excluyentes, pueden coexistir; pero, en este caso, el sistema de mayor complejidad es el que ejerce influencia 
sobre los otros. La influencia es unidireccional: de los más complejos hacia los de menor complejidad, mas no en sentido inverso. Esa influencia puede desembocar en acelerar la transformación de los sistemas menos complejos hacia el sistema tecnológico, pero ello supone, entre otros elementos, una transformación en el tejido empresarial prevaleciente en dichos sistemas.

Esa vinculación entre sistema social y organización empresarial lleva a señalar, primero a nivel de sistema, que: «[...] A mayor complejidad, para la sostenibilidad del sistema se requiere mayor energía social, mayor densidad de energía, alta densidad en la conectividad, y predominio de la energía de cohesión» (p. 37). Mientras que, a nivel de la empresa, dicha vinculación se traduce en afirmar que: «[...] un tipo de empresa denominada $B$ (circular) puede reformular la generación y distribución de energía social, dando origen al fortalecimiento de una energía de cohesión y de mejora de la capacidad adaptativa de la organización y de la sociedad» (p. 11).

\section{De la fragmentación empresarial al sistema de la empresa}

En las páginas iniciales del texto el autor señala: "[...] no hay sociedades sanas sin organizaciones sanas» (p. 7). Dicha frase reitera la interrelación presente entre el nivel micro y macro, aproximación que es transversal a lo largo del libro reseñado.

En este segundo capítulo se pone énfasis en el rol que juegan las empresas como generadoras de energía de cohesión. De esta se dice que "es el resultado de diversos elementos que intervienen en la dinámica de la organización. Uno de estos elementos tiene que ver con la práctica de relaciones y vínculos que se dan en el interior de la organización. El individuo, la organización y el entorno deben estar alineados en la generación de energía social» (p. 51). Se menciona también que, para identificar la energía de cohesión de la empresa, se debe tener claridad sobre el balance de su energía social, si es positivo o negativo. Y para el establecimiento de dicho balance «se deben considerar los aspectos contenidos en las dimensiones política, comunicacional, laboral, económica, ambiental y ética» (p. 51). Se debe realizar la sumatoria de los respectivos saldos de energía social de cada una de las dimensiones señaladas a fin de obtener claridad sobre la continuidad o el conflicto/desorden de la organización. Y ello, trasladado a la esfera del sistema, indicará si es sostenible o va en dirección al colapso.

Al igual que en el caso del sistema, divide las empresas en tres categorías: tradicional, moderna y tecnológica. La primera cuenta con una menor densidad de energía, la moderna cuenta con un poco más que la anterior y la tecnológica dispone de una densidad de energía superior a las categorías previas.

Haciendo uso de conceptos tomados de la geometría, califica el tipo de gestión de dichas categorías de empresas. Señala, así, que la empresa tradicional se caracteriza por un tipo de gestión piramidal; la moderna, por una de tipo rombo; mientras que la tecnológica cuenta con una de corte circular. En relación con las empresas tradicionales, menciona que: "Cuando predominan las energías sociales de las empresas de gestión piramidal, los valores predominantes en la sociedad son: verticalidad, discriminación, imposición, desigualdad, contaminación, paternalismo» (p. 103). Esos valores llevan a un aumento de la entropía y ello conduce a una situación de colapso. 
Por su parte, cuando predomina la energía de cohesión, hay sostenibilidad y esta condición está asociada a las empresas con un modelo gestión circular (empresas B). La sostenibilidad está entendida como «la capacidad de adaptación de un sistema a su entorno cambiante para asegurar su continuidad» (p. 68).

Otro aspecto importante que se desarrolla en este capítulo es la discusión entre valor económico, valor social y valor compartido. Un buen resumen de ella es cuando se escribe que: "Resolver problemas sociales y ambientales haciendo utilidades no es lo mismo que hacer utilidades y resolver dichos problemas» (p. 68). Y más contundente es cuando se afirma que: "La redefinición del concepto de valor nos lleva a plantear que, para la humanidad, la resolución de los problemas sociales y ambientales existentes es lo que debe entenderse por valor» (p. 68)

Pone en cuestión la teoría económica convencional cuando la interpela por concentrar el objetivo de la empresa en la maximización de utilidades; es decir, en la generación solo de valor económico. Esto es clave, ya que desde la raíz se disocia el valor económico del valor social, deslegitimándose la acción empresarial, razón por la cual autores como Porter y Kramer enarbolan el concepto de valor compartido (valor económico y valor social) como el nuevo paradigma que permita un reencuentro de la empresa con la sociedad.

\section{De la responsabilidad social a las empresas $B$}

En el capítulo tercero, Caravedo problematiza el tema de la responsabilidad social empresarial y, en el cuarto, el porqué de la necesidad de que aumente la participación en el Perú, de las empresas B (circulares).

Sobre la responsabilidad social tal como se la practica y se la percibe en el Perú, señala que ella se ha desprestigiado. Su calificativo se basa en el resultado de algunas encuestas de opinión que captan las percepciones poco favorables de los ciudadanos frente a ella y, de otro, expresa su desazón frente a las limitaciones de la implementación de iniciativas diversas calificadas bajo el logo de responsabilidad social empresarial. Indica que en estas la resolución de problemas sociales y ambientales es visto como algo fuera del core de la empresa.

Las empresas B o circulares aparecen como aquellas que concentran la posibilidad de un cambio en la tendencia a la acumulación de energía social y en imprimirle sostenibilidad al sistema. El alcance de ellas ha quedado en evidencia con las referencias en los capítulos anteriores; sin embargo, en el país hay solo cuatro empresas calificadas como empresas B ${ }^{1}$. En consecuencia, la tarea es enorme en lo referido a buscar su expansión sobre los otros dos modelos: el tradicional y el moderno. Las empresas B son depositarias de un modelo de gestión circular con una conectividad más compleja y, por ello, con la capacidad de generar un balance de energía social positivo que agregue cohesión a la empresa y al sistema.

1. Al momento de la redacción final de esta reseña (febrero de 2017), ese número había aumentado a seis. Ver al respecto: <http://elcomercio.pe/economia/negocios/estas-son-seis-empresas-b-certificadas-que-tiene-perunoticia-1944287>. 
bibliografía

\section{Porter, Michael \& Mark Kramer}

2011

La creación de valor compartido. Harvard

Business Review América Latina, enero.

Disponible en: <http://www.

peru2021.org/repositorioaps/0/0/par/

creacionvalorcompartido/shared $\% 20$

value $\% 20 \mathrm{in} \% 20$ spanish.pdf >

Fecha de recepción: 14/11/2016

Fecha de aceptación: 31/01/2017 